Research Paper

\title{
Suppressive Effects of Aspirin for Postthoracotomy Pleural Adhesion in Rats
}

\author{
Shunta Ishihara ${ }^{1}$, Kazuhiro Ito ${ }^{\circledR}{ }^{\bowtie}$, Satoru Okada ${ }^{1}$, Masanori Shimomura ${ }^{1}$, Junichi Shimada ${ }^{1}$, Tadanori \\ Yamaguchi², Masayoshi Inoue ${ }^{1}$ \\ 1. Division of Thoracic Surgery, Department of Surgery, Graduate School of Medical Science, Kyoto Prefectural University of Medicine, 465 Kajii-cho, \\ Kamigyo-ku, Kyoto 602-8566, Japan \\ 2. Department of Cytopathology, Ayabe City Hospital, 20-1 Aono-cho Ootuka, Ayabe 623-0011, Japan
}

$\square$ Corresponding author: Kazuhiro Ito, Division of Thoracic Surgery, Department of Surgery, Graduate School of Medical Science, Kyoto Prefectural University of Medicine, 465 Kaji-cho, Kamigyo-ku, Kyoto 602-8566, Japan. E-mail: kazuitoh@koto.kpu-m.ac.jp; Telephone: +81-75-251-5739; Fax: +81-75-251-5739

(c) Ivyspring International Publisher. This is an open access article distributed under the terms of the Creative Commons Attribution (CC BY-NC) license (https:// creativecommons.org/licenses/by-nc/4.0/). See http://ivyspring.com/terms for full terms and conditions.

Received: 2018.12.28; Accepted: 2019.03.23; Published: 2019.04.25

\begin{abstract}
Background: Postoperative adhesion is one of major concerns at re-thoracotomy. Aspirin has both the anti-platelet and anti-inflammatory effects, and decreases several cytokines production.

Objective: We investigated that aspirin could reduce postoperative adhesion formation in a rat model.

Methods: We cauterised the lung visceral pleural to make postoperative adhesion in rats. The animals were allocated to a control group and an aspirin administration group (100 mg/kg/day for 14 days). We performed re-thoracotomy and evaluated the adhesion lengths on day 14 . We also investigated the cytokine expression in the adhesion region and the peripheral tissue with platelet-derived growth factor (PDGF), platelet-derived growth factor receptor (PDGFR), alpha smooth muscle actin (Q-SMA), transforming growth factor beta 1 (TGF- $\beta 1$ ), and vascular endothelial growth factor-A (VEGF-A), sequentially.

Results: The adhesion lengths were significantly shorter in the aspirin group than that in the control group $(8.7 \pm 2.0 \mathrm{~mm}$ vs $11.2 \pm 1.1 \mathrm{~mm}, p=0.024)$. The expressions of PDGF and PDGFR were lower in the aspirin group than that in the control group on day 3 . The expression of $\alpha$-SMA on fibroblasts decreased in the aspirin group on day 3 . There was no significant difference in the expressions of TGF- $\beta 1$ and VEGF-A with administration of aspirin.

Conclusions: Aspirin could reduce postoperative pleural adhesion by inhibiting the expression of PDGF.
\end{abstract}

Key words: pleural adhesion, reoperation, aspirin, platelet, platelet-derived growth factor

\section{Introduction}

Pleural adhesions, which are often caused by a prior thoracotomy, can make identification of anatomical structures complicated at re-thoracotomy. Re-thoracotomy in a patient with pleural adhesions has several risks of blood loss, pleural injury, and prolonged operative times, and also increases the risk of postoperative complications [1]. Recent reports have shown that surgical resection for metachronous second primary lung cancers improve prognosis $[2,3]$. Repeat metastasectomy is also recommended to improve the survival in patients with metachronous pulmonary metastasis from colorectal carcinoma [4]. Patients who underwent prior pulmonary resections showed evidence of adhesions during 54\% of video-assisted thoracoscopic surgery (VATS) and $100 \%$ of thoracotomies [5]. As such, we sometimes have opportunities to perform pulmonary resections at the time of re-thoracotomy. However, the method for preventing pleural adhesions after thoracotomy remains unclear.

Some anti-adhesion materials are reportedly used in the field of thoracic surgery [6-8]. Previous reports have shown that non-steroidal anti-inflammatory drugs (NSAIDs) or corticosteroids reduced adhesion formation because of their anti-inflammation effects [9-11]. Aspirin, another NSAIDs, may reduce 
adhesions by inhibiting platelet activation through cyclooxygenase (COX) blockade. Platelets release several inflammatory cytokines and activate inflammatory cells, such as neutrophils and macrophages at the sites of injury [12]. These effects are known to play an important role in wound healing. Postoperative adhesions are formed by a local inflammatory response as part of the wound healing process [13]. We hypothesised that aspirin could reduce post-thoracotomy adhesion formation by inhibiting platelet activation.

The purposes of this study were to examine whether daily oral administration of aspirin could reduce the pleural adhesions caused by thoracotomy and to investigate how aspirin affect the histological changes that occur during the period of pleural adhesion formation.

\section{Methods}

All animal experiments were conducted with the approval of, and in accordance with the guidelines of the Committee for Animal Research at Kyoto Prefectural University of Medicine (approval number:
M27-435, M28-435).

\section{Experimental design}

Nine-week-old male Sprague-Dawley rats (325 $\pm 15 \mathrm{~g}$ ) were purchased from CLEA Japan (Osaka, Japan). They were housed in a controlled environment for one week before starting the experiment and were allowed free access to water and a maintenance diet. Aspirin (Lot No. A2093) was purchased from Sigma-Aldrich Japan (Tokyo, Japan). Distilled water was purchased from Otsuka (Tokyo, Japan).

Animals were randomly divided into two groups after the first operation, the aspirin group $(n=8)$ and the control group $(n=8)$. Aspirin was suspended in a $0.3 \%$ carboxymethylcellulose solution. The aspirin solution was adjusted to a concentration of $16.5 \mathrm{mg} / \mathrm{ml} .2 \mathrm{ml}$ of the aspirin solution (100 $\mathrm{mg} / \mathrm{kg} /$ day) was administrated orally to rats in the aspirin group, and the same volume of distilled water was administered to rats in the control group. The animals underwent re-thoracotomy, and the adhesion lengths were evaluated 14 days after the initial operation (Fig 1A).

A

\section{Cauterizing Procedure}

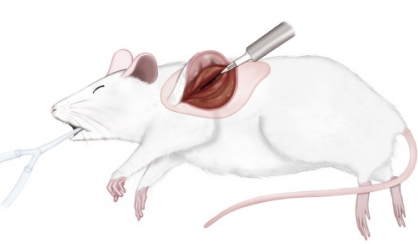

Male 10 weeks SD rat

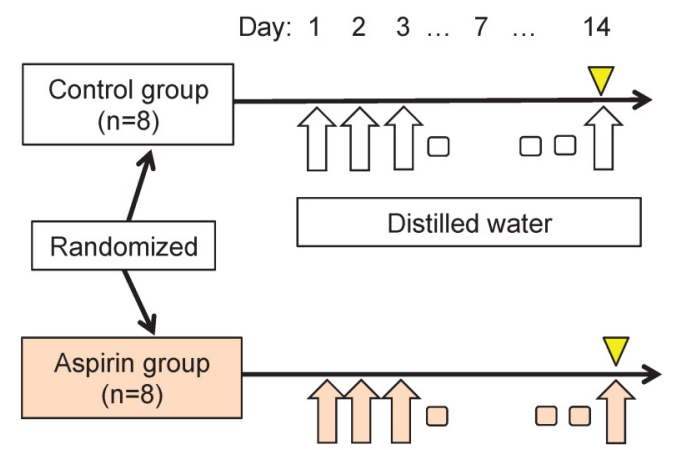

Aspirin (100 mg $/ \mathrm{kg} /$ day)
2. Re-thoracotomy

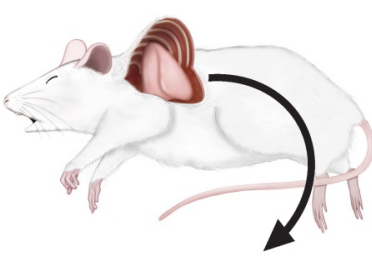

3. Measure adhesion length

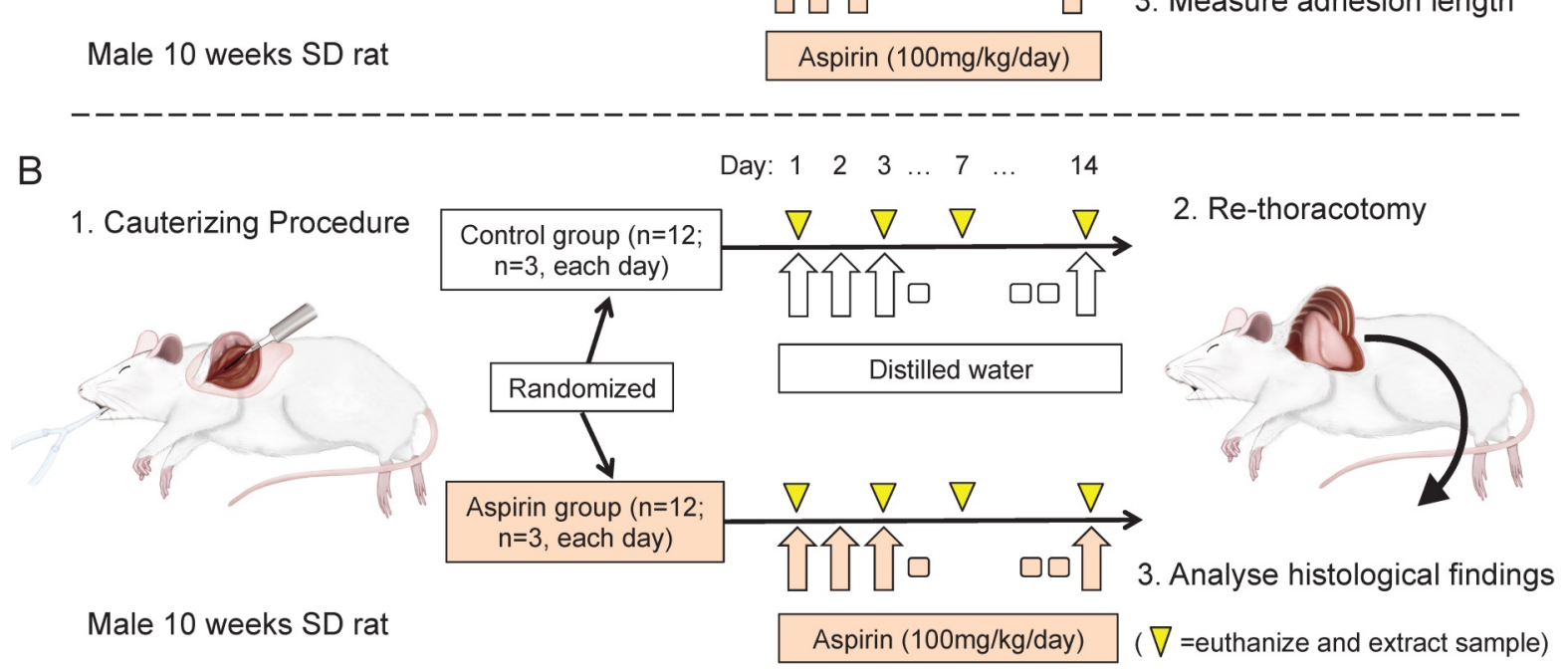

Figure 1. Experimental design: (A) Sprague-Dawley rats underwent thoracotomy and cauterisation to the visceral pleura to induce adhesion. They were randomly allocated to the aspirin group $(n=8)$ or the control group $(n=8)$, and received aspirin or distilled water, respectively. After 14 days, re-thoracotomy was performed, and the adhesion length was measured. (B) Animals underwent the same surgical procedure to cause adhesion and were administered with aspirin or distilled water, respectively ( $n=12$, each group). On days $1,3,7$ and 14 after the procedure, the samples $(n=3$, each day) were extracted, and were analysed for histological examination. 
Another series of the experiment was performed to histological examination of the adhesion formation sequentially. Rats were undergone the same surgical procedure to make adhesions and was administered aspirin or distilled water $(n=12$, each group). The animals were euthanized by the administration of pentobarbital and the chest wall samples were extracted on days $1,3,7$, and $14(n=3$, each day, Fig 1B).

\section{Surgical technique}

Anaesthesia was induced by inhalation of isoflurane (WAKENYAKU CO., Kyoto, Japan) and was maintained with an intraperitoneal injection of pentobarbital (35 mg/kg, WAKENYAKU CO.). A 16-gauge angiocatheter was inserted into the trachea, and the animals were mechanically ventilated at 3 $\mathrm{ml} \times 30$ breaths / $\mathrm{min}$ with a positive end-expiratory pressure of $2 \mathrm{cmH}_{2} \mathrm{O}$.

A left lateral thoracotomy in the fourth intercostal space was performed. The length of the intercostal incision was $20 \mathrm{~mm}$. The visceral pleura of the length of $10 \mathrm{~mm}$ beneath the intercostal incision was cauterised using a high-temperature cautery devise (Bovie Medical, Clearwater, FL, USA) for 30 seconds (Fig 2A). The animals were euthanised by blood loss after the intraperitoneal administration of pentobarbital $(65 \mathrm{mg} / \mathrm{kg})$.

We extirpated the entire samples of the left lung adhering to the chest wall from the second to the seventh intercostal spaces after redo-thoracotomy. The length of the pleural adhesions was measured at the caudal side using a digital calliper in blinded fashion (Fig 2B).

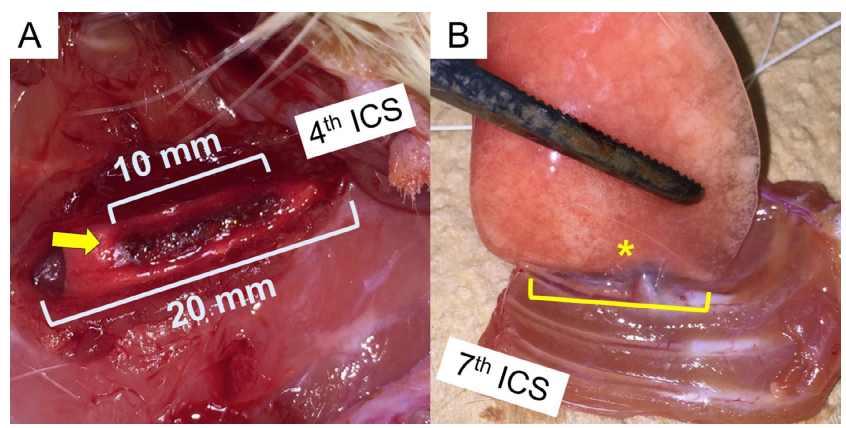

Figure 2. Surgical technique: (A) Left lateral thoracotomy through the 4th Intercostal space and cauterisation of the visceral pleura were performed (arrow). The intercostal incision length and cauterising length are $20 \mathrm{~mm}$ and 10 $\mathrm{mm}$, respectively. (B) After the study period, the whole left lung and chest wall with 2-7th ribs were extirpated. The length of adhesion (asterisk) between the lung and chest wall was measured with a digital calliper.

\section{Evaluation of the histology of adhesion formation}

The extracted samples for histological evaluation on day $1,3,7$, and $14(\mathrm{n}=3$, each days) were fixed in a
$10 \%$ formaldehyde aqueous solution. The fixed specimens were vertically cut in the midline of the pleural adhesion and embedded in paraffin blocks. The sections were cut $4-\mu \mathrm{m}$ thick and evaluated after haematoxylin-eosin (HE) staining. Antigen retrieval was performed with a citrate acid buffer. The immunohistochemical staining analysis was performed using platelet derived growth factor A (PDGF-A, 1:100, mouse monoclonal, sc-9974, Santa Cruz Biotechnology, Santa Cruz, CA, USA), platelet derived growth factor B (PDGF-B, 1:200, rabbit polyclonal, ab16829, Abcam, Cambridge, MA, USA), platelet derived growth factor receptor alpha (PDGFR-a, 1:200, rabbit polyclonal, ab5460, Abcam), alpha smooth muscle actin (a-SMA, 1:200, rabbit polyclonal, ab5694, Abcam), transforming growth factor beta 1 (TGF- $\beta 1,1: 200$, rabbit polyclonal, ab92486, Abcam), and vascular endothelial growth factor A (VEGF-A, 1:200, mouse monoclonal, ab1316, Abcam). All sections were coloured by 3,3'-diaminobenzidine for 10 minutes.

Expressions of the cytokine staining were divided into four intensity grades (no staining, mild staining, moderate staining, and intense staining) in the adhesion region and along the border of the normal lung as previously reported [14]. The intensity grade was judged using the majority samples of the grade in each immunohistochemical stainings.

The number of macrophages expressing the PDGF-A, PDGF-B and PDGFR- $\alpha$ and the number of fibroblast expressing the a-SMA were counted in the adhesion region and the border of the normal lung (Figure 3A-D). The manual cell counts expressing the cytokines were divided by the cauterised area, in order to standardise. We manually doted the cauterised region and calculated the area by using image J software (1.51s, National Institutes of Health, Bethesda, MD, USA) [15] (Figure 3E).

\section{Statistical analysis}

Statistical analysis was performed using JMP software (version 12.2; SAS Institute Inc., Cary, NC, USA). All data were analysed with the MannWhitney's U test. Two-way ANOVA was used for the comparisons of two groups among each day. A $p$-value $<0.05$ was considered statistically significant. The results were described as the mean \pm standard deviation.

\section{Results}

\section{Adhesion length}

One sample in the control group was excluded because of rat death in the administration period from an unknown cause. Two samples in the aspirin group 
were excluded because of total absence of the pleural adhesions. The adhesion length was significantly shorter in the aspirin group $(n=6)$ than that in the control group $(\mathrm{n}=7)(8.7 \pm 2.0 \mathrm{~mm}$ vs. $11.2 \pm 1.0 \mathrm{~mm}$, respectively; $p=0.024$ ) (Fig 4 ).

\section{Histology of the adhesion formation}

Macroscopically, fibrin clots and blood piles surrounded the injured section on day 1 after the procedure, and no adhesion was observed. On day 3 , loose adhesions easily dissected were observed between the injured lung and the chest wall. On day 7, adhesions completed along the line of the thoracotomy incision and were firmly formed on day 14.

On day 1, fibrin and blood clots were piled in the control group. Inflammatory cells such as neutrophils and macrophages infiltrated into the surface of the injured lung and the along the region bordering the normal lung (Fig 5A, E). On day 3, the fibrin clot was decreased and many inflammatory cells were infiltrating into the clot. The inflammatory cells increased on the injured lung surface and in the region bordering the normal lung. Fibroblasts appeared and some tiny vessels were beginning to appear in the region bordering the normal lung (Fig 5B, F). On day 7, the fibrin clot had almost disappeared, and the adhesion had completed between the chest wall and the lung. In the adhesion area, the fibroblasts increased and the density of the infiltrating inflammatory cells decreased. The vascularity increased at the site bordering the normal lung and in the area of the adhesions (Fig 5C, G). On day 14 , the density of the fibroblasts was increasing in the adhesion area. Vascular endothelial cells were proliferating and the matured vessels were observed in the adhesion area (Fig 5D, H).

During the adhesion formation process, the time course of macroscopic and histological findings of the aspirin group was morphologically similar to that of the control group. Inflammatory cells emerged and fibrin and blood clots were piled from day 1 . On day 3 , the fibrin clot shrunk and the inflammatory cells migrated. Fibroblasts appeared in this period as with the control group. The adhesion had completed on day 7 and had formed densely with fibroblasts and vessels increasingly on day 14 (Fig 5I-L).

\section{Immunohistochemical evaluation of the adhesion site}

The time course of recruited inflammatory cells and cytokines in the adhesion site of the lung is shown in Table 1 and Figure 6A-X.

Table 1. Time course of recruited inflammatory cells and cytokines at the adhesion site of the lung

\begin{tabular}{|c|c|c|c|}
\hline Cytokine & $\begin{array}{l}\text { Peak of the } \\
\text { expression }\end{array}$ & $\begin{array}{l}\text { Expressing } \\
\text { cells }\end{array}$ & Site of expression \\
\hline PDGF-A & Day 3 & $\begin{array}{l}\text { Neutrophils } \\
\text { Macrophages }\end{array}$ & $\begin{array}{l}\text { Adhesion site or injured lung layer } \\
\text { The region bordering normal lung } \\
\text { Fibrin clot }\end{array}$ \\
\hline PDGF-B & Day 1 & $\begin{array}{l}\text { Macrophages } \\
\text { Fibroblasts }\end{array}$ & $\begin{array}{l}\text { Adhesion site or injured lung layer } \\
\text { Fibrin clot }\end{array}$ \\
\hline PDGFR-a & Day 3 & $\begin{array}{l}\text { Neutrophils } \\
\text { Macrophages }\end{array}$ & $\begin{array}{l}\text { Adhesion site or injured lung layer } \\
\text { The region bordering normal lung }\end{array}$ \\
\hline a-SMA & Day 3 & $\begin{array}{l}\text { Fibroblasts } \\
\text { Neutrophils }\end{array}$ & The region bordering normal lung \\
\hline TGF- $\beta$ & Day 1-3 & $\begin{array}{l}\text { Neutrophils } \\
\text { Macrophages } \\
\text { Fibroblasts }\end{array}$ & $\begin{array}{l}\text { Adhesion site or injured lung layer } \\
\text { The region bordering normal lung } \\
\text { Fibrin clot }\end{array}$ \\
\hline VEGF-A & Day 1-3 & Neutrophils & $\begin{array}{l}\text { Adhesion site or injured lung layer } \\
\text { The region bordering normal lung } \\
\text { Fibrin clot }\end{array}$ \\
\hline
\end{tabular}

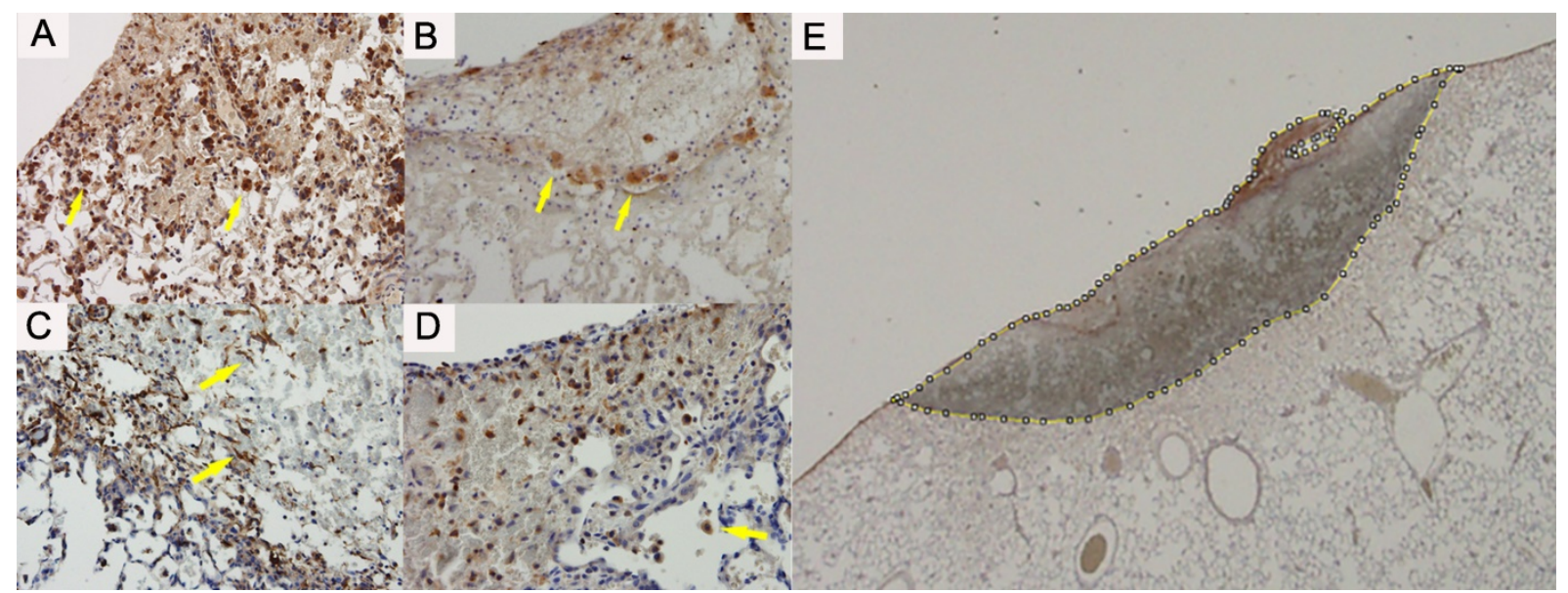

Figure 3. (A) Platelet-derived growth factor A expressed on the macrophages (arrow) and neutrophils. (B) Platelet-derived growth factor B expressed clearly on the macrophages (arrow). (C) Platelet-derived growth factor Receptor alpha expressed on the macrophages (arrow) and neutrophils. (D) Alpha smooth muscle actin expressed mainly on the fibroblast on the border of the injured lung (arrow). (E) The slides of immunohistochemical staining are captured as digital data. We manually doted the cauterised region and calculated the area using image J software. The counted cells (arrows) were divided by the adhesion area of each section. 
PDGF-A, PDGF-B and PDGFR- $\alpha$ expressions were observed from the day 1 (Fig 6A, E, I). The strongest expression was found on day 3 (Fig 6B, F, J). The staining intensity of PDGF-A on day 1 and those of PDGF-A, PDGF-B and PDGFR-a on day 3 were weaker in the aspirin group than those in the control group (Fig 7A-H).

a-SMA expression in the control group was observed from the day 3 (Fig 6N). The staining intensity was weaker in the aspirin group than that in the control group on day 3. TGF- $\beta 1$ and VEGF-A expressions in the control group were also observed from day 1 (Fig 6Q, U), and they were strongest on day 3 (Fig 6R, V). The intensity was almost similar to that in the aspirin group.

On day 1, numerous macrophages expressing PDGF-B were found. The amount of PDGF-B expressed on macrophages in the aspirin group expressed was less than that in the control group on day 1 (Fig 8B). We observed numerous macrophages expressing PDGF A and PDGFR-a on day 3, and the number of macrophages expressing PDGF-A and PDGFR- $\alpha$ were less in the aspirin group than that in the control group on the 3rd (Fig 8A, C). The a-SMA expressed on the fibroblasts at the lung border on day 3 in the control group. The number of fibroblasts expressing a-SMA was less in the aspirin group than that in the control group on day 3 (Fig 8D).

\section{Discussion}

In this study, we showed that aspirin suppressed the development of postoperative pleural adhesions in rats. Several studies have reported the inhibition of pleural adhesions in animal models [8, 16-18]. In those studies, pleural adhesions were reproduced using a scratching method [16, 17] or cauterization of the pleura $[8,18]$. We opted to cauterize the pleura for pleurodesis, because it was the most useful technique for the quantitative method of evaluating pleural adhesions that we designed. We cauterized the visceral pleura through the intercostal space at a fixed distance, and the pleural adhesions were formed according to the surgical treatment in the control group.

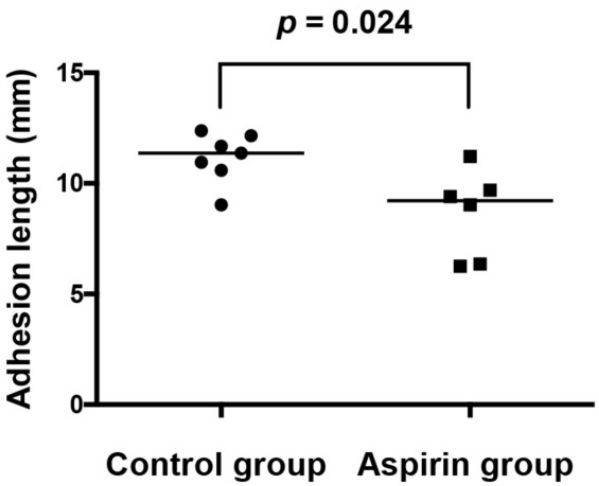

Figure 4. The adhesion length is significantly shorter in the aspirin group than that in the control group $(8.7 \pm 2.0 \mathrm{~mm}$ vs. $11.2 \pm 1.1 \mathrm{~mm})$.

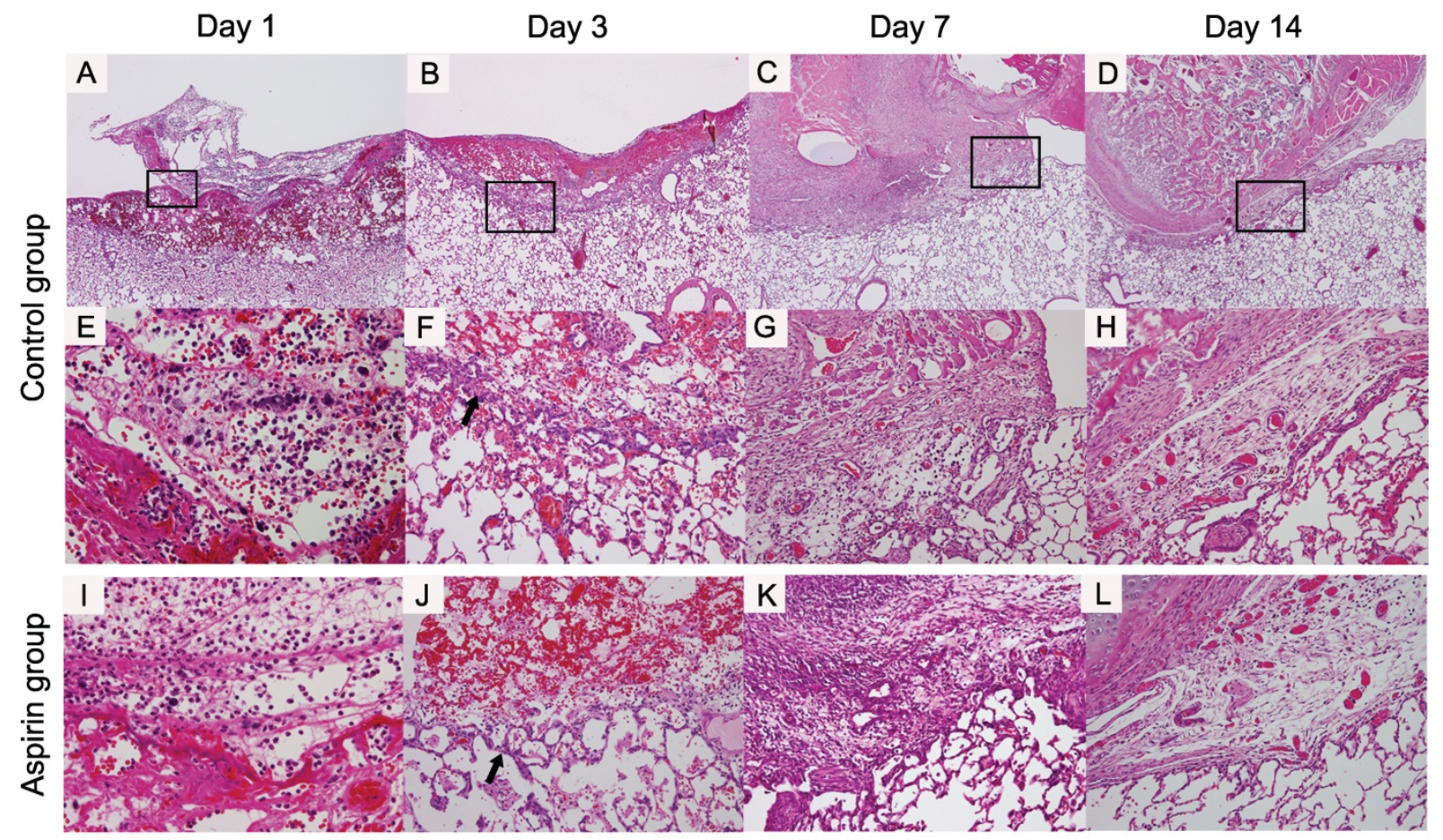

Figure 5. Histological findings around the procedure site of the lung (original magnification $\times 40 ; \mathrm{A}-\mathrm{D}, \times 400 ; \mathrm{E}, \times 200$; F-H): In the control group, on day 1, the fibrin clots and red blood cells are piled (A, E). On day 3, the fibroblast (arrow) appear in the border between the procedure site and normal pulmonary parenchyma of the lung border (B, F). On day 7, the adhesion between the chest wall and the lung is formed entirely (C, G). On day 14, the fibroblasts are increased in number and the adhesion became dense $(D, H)$. In aspirin group, these findings were morphologically similar the control group $(\times 400 ; \mathrm{I}, \times 200 ; \mathrm{J}-\mathrm{L})$. 


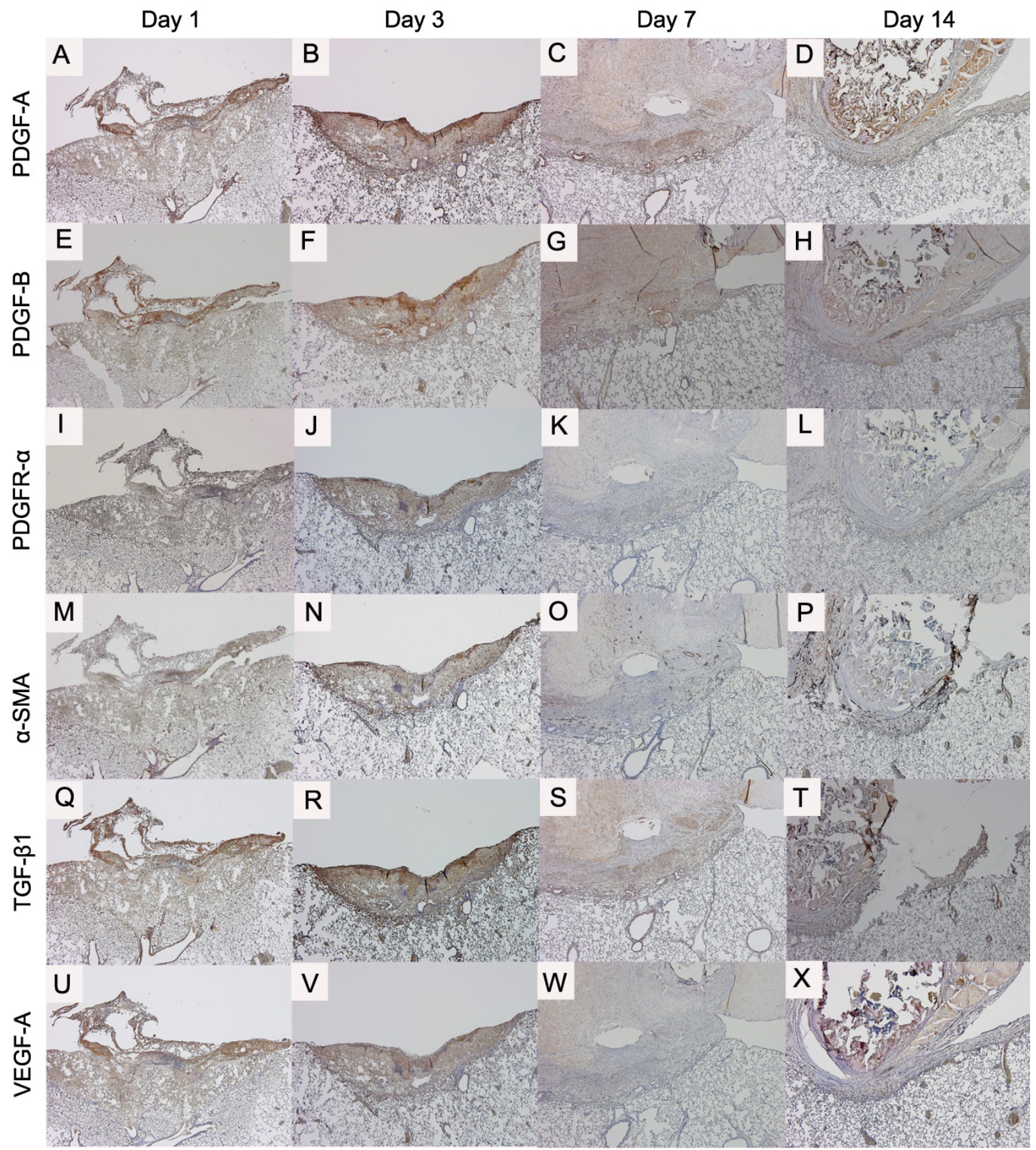

Figure 6. Time course of the expression of cytokines in the control group (original magnification $\times 40$ ): platelet-derived growth factor A (PDGF-A) (A-D); platelet-derived growth factor B (PDGF-B) (E-H); platelet-derived growth factor receptor alpha (PDGFR- $\alpha$ ) (I-L); and alpha smooth muscle actin ( $\alpha-S M A)$ (M-P); transforming growth factor beta 1 (TGF- $\beta 1)(\mathrm{Q}-\mathrm{T})$; and vascular endothelial growth factor A (VEGF-A) (U-X).

Through our experiments, we demonstrated the immunohistochemical expression of PDGFs and that an increasing number of macrophages expressed PDGFs on days 1 and 3. To the best of our knowledge, PDGF has never before been reported to associate with adhesion formation. We showed that PDGFs potentially affected pleural adhesion formation in the early phase. PDGF is released by platelets and secreted by activated macrophages at the site of injury during the wound healing process [19]. We showed that PDGFs were expressed on the inflammatory cells during the adhesion process.

We showed that pleural adhesions were formed during the wound healing process. Cytokines PDGF-A, PDGF-B, PDGFR- $\alpha$, TGF- $\beta 1$, and VEGF-A were expressed on day 1 after the procedure, and the expression of most of these cytokines peaked on day 3. These results are compatible with those of previous studies reporting that these cytokines function mainly in the early phases of wound healing [20-22]. These cytokines were shown to be relevant to pleural adhesion formation in the early phase. 
PDGF expression and the number of macrophages expressing PDGFs decreased in the aspirin group compared with that in the control the group. Moreover, the number of fibroblasts expressing a-SMA decreased in the aspirin group. Hence, aspirin could inhibit PDGF secretion, inhibit macrophage activation, and inhibit fibroblast recruitment. Aspirin exhibits anti-inflammatory effects and inhibits platelet activation by blocking the production of thromboxane A2 [23]. These interactions could supress the production of several cytokines, including PDGFs, thus preventing the congregation of inflammatory cells. Aspirin also directly inhibits macrophage activation [24, 25]. Our observations might be explained by these mechanisms.

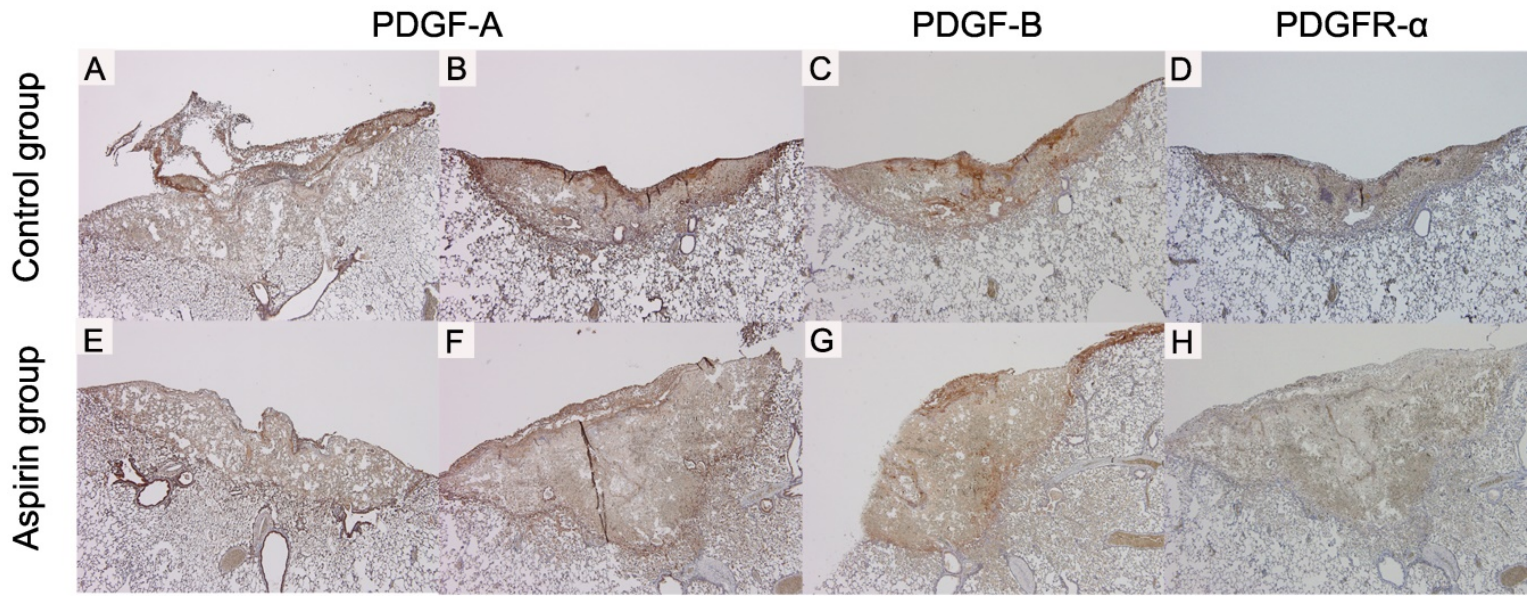

Figure 7. The immunohistochemical staining shows difference between control group and aspirin group (original magnification $\times 40$ ): platelet-derived growth factor A (PDGF-A) expression on day 1 in the control $(A,=$ moderate) and in the aspirin group $(E,=$ mild $)$, and on day 3 in the control $(B,=$ strong) and in the aspirin group $(F$, =moderate); platelet-derived growth factor B (PDGF-B) expression on day 3 in the control (C, =strong) and in the aspirin group (G, =moderate); and platelet-derived growth factor receptor alpha (PDGFR- $\alpha$ ) expression on day 3 in the control $(\mathrm{D},=$ strong) and in the aspirin group $(\mathrm{H},=$ moderate).
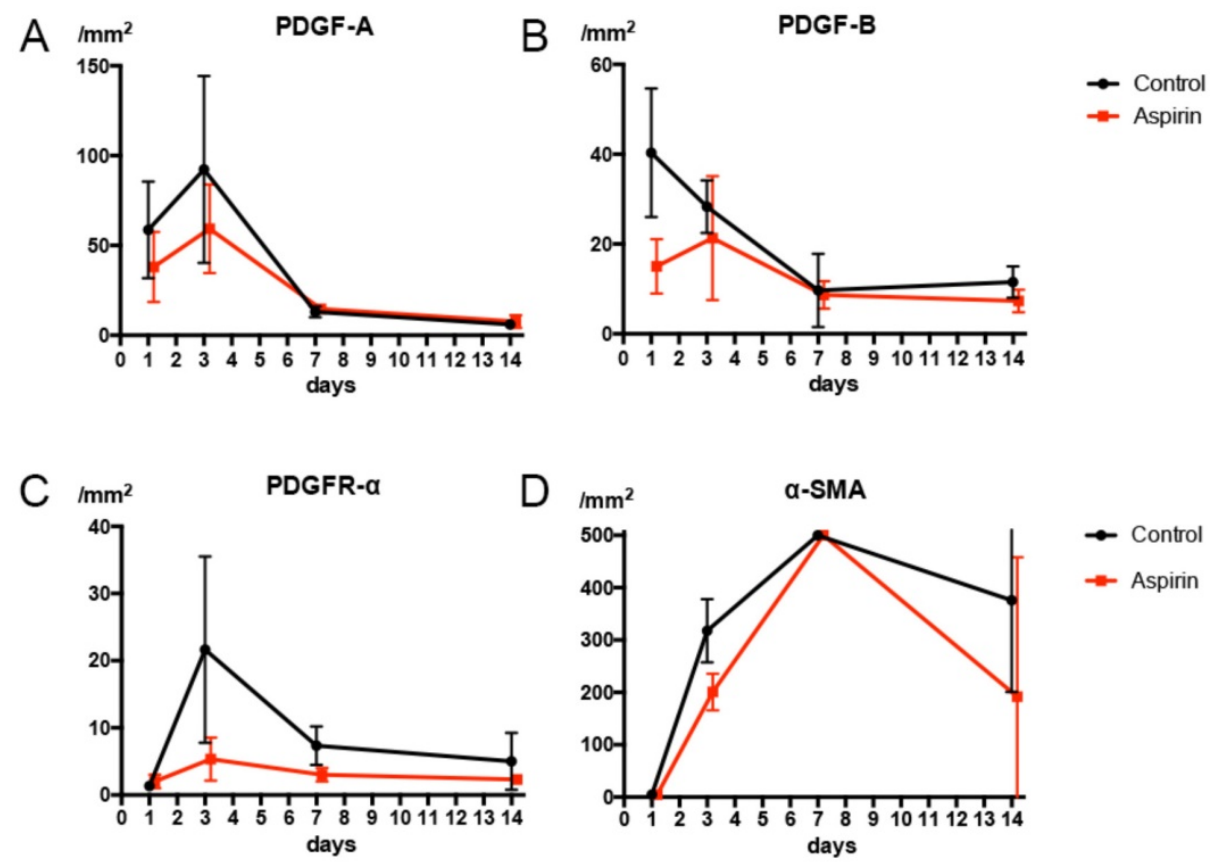

Figure 8. (A) The number of macrophages expressing platelet-derived growth factor A (PDGF-A) is less in the aspirin group on day 1. (B) The number of macrophages expressing platelet-derived growth factor B (PDGF-B) is less in the aspirin group on day 1 . (C) The number of macrophages expressing platelet-derived growth facto receptor alpha (PDGFR- $\alpha$ ) is less in the aspirin group on day 3. (D) The number of fibroblasts expressing alpha smooth muscle actin ( $\alpha-S M A)$ is less in the aspirin group on day 3 .

TGF- $\beta 1$ is known to be increased in sites of adhesion [26]. Similar to PDGFs, TGF- $\beta 1$ is abundant in the platelet-rich plasma [20]. Acetylsalicylic acid inhibits cell proliferation through TGF- $\beta$ expression
[27]. However, in this study, we observed no difference in TGF- $\beta$ expressions between the aspirin and control groups.

VEGF are released in the early phase of 
postoperative adhesions development [28]. Because VEGFs are not abundant in platelet-rich plasma, aspirin might not be highly effective in reducing VEGF. This may be why, with respect to VEGF expression, we observed no difference between the aspirin and control groups.

This study has two limitations. First, we set the period for adhesion formation to 14 days, because platelets begin to secrete activating factors during the early phases after injury [22]. In a previous report, mesothelial cells began to emerge at 3-5 days after injury to the pleural surface [29], and the adhesions were formed by day 7 after pleurodesis [18]. In our study, most dynamic cytokine interactions were observed on days 1 and 3 after the surgical procedure. The pleural adhesions were already present by the 7th day and showed histopathological changes up to 14 days after pleurodesis. Our results showed that aspirin could suppress pleural adhesion formation in the early phase; however, these effects were not observed in the chronic phase of adhesion development (beyond 14 days). Second, we administrated high-dose aspirin to rats. The dose-threshold for aspirin in rats has not been sufficiently investigated. Previous reports in small animals showed that high-dose aspirin inhibited alveolar COX-1 and -2 [30] or arterial thrombosis [31]. We selected high-dose aspirin to inhibit pleural adhesions formation in a rat model; however, the effect of low-dose aspirin was not investigated.

\section{Conclusion}

Aspirin inhibited pleural adhesion formation in rats via decreased PDGF expression, followed by suppression of macrophage fibroblast infiltration.

\section{Acknowledgment}

The authors would like to thank Editage (www.editage.jp) for English language editing.

\section{Funding}

This work was supported by Japan Society for the Promotion of Science (JSPS) KAKENHI, Grant Number J15K11943.

\section{Authors' contributions}

S.I. conceptualised the study. K.I. and S.O. lead to plan the study. S.I. and S.O. collected the samples and analysed the data. T.Y. prepared the specimens. S.I. drafted the article; K.I., S.O., M.S., J.S., and M.I. revised the article critically. All authors approved the article.

\section{Competing Interests}

The authors have declared that no competing interest exists.

\section{References}

1. Yim AP, Liu HP, Hazelrigg SR, Izzat MB, Fung AL, Boley $T M$, et al. Thoracoscopic operations on reoperated chests. Ann Thorac Surg. 1998; 65: 328-30.

2. Yang J, Liu M, Fan J, Song N, He WX, Yang YL, et al. Surgical treatment of metachronous second primary lung cancer. Ann Thorac Surg. 2014; 98: 1192-8.

3. Muranishi Y, Sonobe M, Hamaji M, Kawaguchi A, Hijiya K, Motoyama H, et al. Surgery for metachronous second primary lung cancer versus surgery for primary lung cancer: a propensity score-matched comparison of postoperative complications and survival outcomes. Interactive cardiovascular and thoracic surgery. 2018; 26: 631-7.

4. Park JS, Kim HK, Choi YS, Kim K, Shim YM, Jo J, et al. Outcomes after repeated resection for recurrent pulmonary metastases from colorectal cancer. Annals of oncology : official journal of the European Society for Medical Oncology. 2010; 21: 1285-9.

5. Tanaka K, Hida Y, Kaga K, Kato H, lizuka M, Cho Y, et al. Video-assisted thoracoscopic surgery lowers the incidence of adhesion to the chest wall but not to the mediastinal and interlobar pleurae. Surgical laparoscopy, endoscopy \& percutaneous techniques. 2010; 20: 46-8.

6. Zhou J, Liwski RS, Elson C, Lee TD. Reduction in postsurgical adhesion formation after cardiac surgery in a rabbit model using N,O-carboxymethyl chitosan to block cell adherence. The Journal of thoracic and cardiovascular surgery. 2008; 135: 777-83.

7. Getman V, Devyatko E, Wolner E, Aharinejad S, Mueller MR. Fleece bound sealing prevents pleural adhesions. Interactive cardiovascular and thoracic surgery. 2006; 5: 243-6.

8. Takagi K, Tsuchiya T, Araki M, Yamasaki N, Nagayasu T, Hyon SH, et al. Novel biodegradable powder for preventing postoperative pleural adhesion. The Journal of surgical research. 2013; 179: e13-9.

9. Lardinois D, Vogt P, Yang L, Hegyi I, Baslam M, Weder W. Non-steroidal anti-inflammatory drugs decrease the quality of pleurodesis after mechanical pleural abrasion. European journal of cardio-thoracic surgery : official journal of the European Association for Cardio-thoracic Surgery. 2004; 25: 865-71.

10. Xie C, Teixeira LR, McGovern JP, Light RW. Systemic corticosteroids decrease the effectiveness of talc pleurodesis. American journal of respiratory and critical care medicine. 1998; 157: 1441-4.

11. Teixeira LR, Wu W, Chang DS, Light RW. The effect of corticosteroids on pleurodesis induced by doxycycline in rabbits. Chest. 2002; 121: 216-9.

12. Singer AJ, Clark RA. Cutaneous wound healing. The New England journal of medicine. 1999; 341: 738-46.

13. Hellebrekers BW, Kooistra T. Pathogenesis of postoperative adhesion formation. The British journal of surgery. 2011; 98: 1503-16.

14. Akyildiz H, Akcan A, Sozuer E, Kucuk C, Yilmaz N, Deniz K. The preventive effect of Met-RANTES on postoperative intraperitoneal adhesion formation in the rat model. Surgery. 2008; 144: 404-9.

15. Schneider CA, Rasband WS, Eliceiri KW. NIH Image to ImageJ: 25 years of image analysis. Nature methods. 2012; 9: 671-5.

16. Tanaka A, Abe T, Matsuura A. Prevention of postoperative intrapleural adhesion of the thoracotomy incision by a bioresorbable membrane in the rat adhesion model. Annals of thoracic and cardiovascular surgery : official journal of the Association of Thoracic and Cardiovascular Surgeons of Asia. 2000; 6: 151-60.

17. Karacam V, Onen A, Sanli A, Gurel D, Kargi A, Karapolat S, et al. Prevention of pleural adhesions using a membrane containing polyethylene glycol in rats. International journal of medical sciences. 2011; 8: 380-6.

18. Akerberg D, Posaric-Bauden M, Isaksson $\mathrm{K}$, Andersson R, Tingstedt B. Prevention of pleural adhesions by bioactive polypeptides - a pilot study. International journal of medical sciences. 2013; 10: 1720-6.

19. Heldin $\mathrm{CH}$, Westermark B. Mechanism of action and in vivo role of platelet-derived growth factor. Physiological reviews. 1999; 79: 1283-316.

20. Burnouf T, Strunk D, Koh MB, Schallmoser K. Human platelet lysate: Replacing fetal bovine serum as a gold standard for human cell propagation? Biomaterials. 2016; 76: 371-87.

21. Golebiewska EM, Poole AW. Platelet secretion: From haemostasis to wound healing and beyond. Blood reviews. 2015; 29: 153-62.

22. Fernandez-Moure JS, Van Eps JL, Cabrera FJ, Barbosa Z, Medrano Del Rosal G, Weiner BK, et al. Platelet-rich plasma: a biomimetic approach to enhancement of surgical wound healing. The Journal of surgical research. 2017; 207: 33-44.

23. Fuster V, Sweeny JM. Aspirin: a historical and contemporary therapeutic overview. Circulation. 2011; 123: 768-78.

24. Liu Y, Fang S, Li X, Feng J, Du J, Guo L, et al. Aspirin inhibits LPS-induced macrophage activation via the NF-kappaB pathway. Scientific reports. 2017; 7: 11549.

25. Malvezi AD, da Silva RV, Panis C, Yamauchi LM, Lovo-Martins MI, Zanluqui NG, et al. Aspirin modulates innate inflammatory response and inhibits the entry of Trypanosoma cruzi in mouse peritoneal macrophages. Mediators of inflammation. 2014; 2014: 580919. 
26. Krause TJ, Katz D, Wheeler CJ, Ebner S, McKinnon RD. Increased levels of surgical adhesions in TGFbeta1 heterozygous mice. Journal of investigative surgery : the official journal of the Academy of Surgical Research. 1999; 12: 31-8.

27. Redondo S, Santos-Gallego CG, Ganado P, Garcia M, Rico L, Del Rio M, et al. Acetylsalicylic acid inhibits cell proliferation by involving transforming growth factor-beta. Circulation. 2003; 107: 626-9.

28. Cahill RA, Wang JH, Soohkai S, Redmond HP. Mast cells facilitate local VEGF release as an early event in the pathogenesis of postoperative peritoneal adhesions. Surgery. 2006; 140: 108-12.

29. Noishiki Y, Shintani N. Anti-adhesive membrane for pleural cavity. Artificial organs. 2010; 34: 224-9.

30. Kirkby NS, Chan MV, Lundberg MH, Massey KA, Edmands WM, MacKenzie LS, et al. Aspirin-triggered 15-epi-lipoxin A4 predicts cyclooxygenase-2 in the lungs of LPS-treated mice but not in the circulation: implications for a clinical test. FASEB journal : official publication of the Federation of American Societies for Experimental Biology. 2013; 27: 3938-46.

31. Konishi N, Hiroe K, Kawamura M. Synergistic effect of a factor Xa inhibitor, TAK-442, and antiplatelet agents on whole blood coagulation and arterial thrombosis in rats. Thrombosis research. 2010; 126: 124-9. 